\title{
Permanent Pacemaker Implantation in Patients With Isolated Persistent Left Superior Vena Cava From a Right- Sided Approach: Technical Considerations and Follow-Up Outcome
}

\author{
Santosh Kumar Sinha ${ }^{\mathrm{a}, \mathrm{b}}$, Amit Goel ${ }^{\mathrm{a}}$, Mahmodula Razia ${ }^{\mathrm{a}}$, Mukesh Jitendra Jha ${ }^{\mathrm{a}}$, \\ Vikas Mishra $^{\text {a }}$, Puneet Aggarwaal ${ }^{\mathrm{a}}$, Ramesh Thakur ${ }^{\mathrm{a}}$, Vinay Krishna ${ }^{\mathrm{a}}$, \\ Umeshwar Pandey ${ }^{\mathrm{a}}$, Chandra Mohan Varma ${ }^{\mathrm{a}}$
}

\begin{abstract}
Background: Positioning a permanent pacing wire in patients with persistent left superior vena cava (PLSVC) to right ventricle often comes as on-table surprise. It is technically demanding and therefore most of operators prefer left-sided approach. We assessed technical challenges during pacemaker implantation, and their short- and longterm outcomes among patients with isolated PLSVC from a rightsided approach.
\end{abstract}

Methods: Thirty-one consecutive patients with isolated PLSVC and 93 patients with right superior vena cava (RSVC) were enrolled with syncope with sinus node dysfunction (SND) and atrioventricular (AV) block. Study was designed on the basis of nested case-control method, and therefore 1:3 proportions was the enrolment criteria to detect any difference as statistically significant as incidence of isolated PLSVC is low.

Results: Mean age of patients was $64.8 \pm 10.5$ years. SND was the most common indication $(\mathrm{n}=55 ; 44 \%)$ followed by AV block $(\mathrm{n}=$ $47 ; 37 \%)$. Nineteen $(20 \%)$ patients received tined pacing lead, while $105(85 \%)$ had screwing lead. There was no significant difference in mean procedural time ( $25 \pm 11$ min vs. $23 \pm 12 \mathrm{~min} ; \mathrm{P}=0.24)$, mean fluoroscopic time $(3.1 \pm 2.2 \mathrm{~min}$ vs. $2.7 \pm 2.1 \mathrm{~min} ; \mathrm{P}=0.54)$, pacing parameters for atrial and ventricular leads, dislodgement rate $(3.2 \%$ vs. $4.8 \% ; \mathrm{P}=0.32)$ and follow-up duration $(6.9 \pm 1.3$ years vs. $7.2 \pm$ 1.1 years; $\mathrm{P}=0.18$ ) between two groups. Compared to patients with RSVC, those with PLSVC had alpha loop configuration for ventricular lead which was statistically significant ( 31 vs. $00 ; \mathrm{P}=0.002$ ).

Conclusions: Patients with PLSVC had alpha loop configuration for

Manuscript submitted September 30, 2018, accepted January 10, 2019

aDepartment of Cardiology, LPS Institute of Cardiology, G.S.V.M. Medical College, Kanpur, Uttar Pradesh, India

${ }^{b}$ Corresponding Author: Santosh Kumar Sinha, Department of Cardiology, LPS Institute of Cardiology, G.S.V.M. Medical College, Kanpur, Uttar Pradesh 208002, India. Email: fionasan@rediffmail.com

doi: https://doi.org/10.14740/cr784 ventricular lead because of circuitous course via left mediastinum. Although pacemaker implantation through coronary sinus via isolated PLSVC from right sided-approach is technically challenging, it obtains good long-term results but needs frequent follow-up during the initial period.

Keywords: Alpha loop configuration; Coronary sinus; Lead dislodgement; Permanent pacemaker implantation; Persistent left superior vena cava

\section{Introduction}

Persistent left superior vena cava (PLSVC), the most common congenital malformation of the thoracic venous system with an incidence of $0.3-0.5 \%$ is generally asymptomatic [1]. In $60 \%$ of the cases, a right superior vena cava (RSVC) is also present and PLSVC and RSVC are connected by the left brachiocephalic vein. In $20 \%$ of cases, RSVC is absent resulting in drainage of venous blood from the upper extremities through left brachiocephalic vein, PLSVC and coronary sinus (CS) into the right atrium, which is also known as isolated PLSVC [2, $3]$. It is an exceedingly rare anomaly, occurring in $0.07-0.13 \%$ of patients who have congenital heart defects such as atrial septal defect, bicuspid aortic valves, coarctation of the aorta, left isomerism or cor triatrium [4]. These venous anomalies are usually asymptomatic and discovered incidentally during imaging and the aberrant position of a pacing lead, central venous catheters, or retrograde cardioplegia for cardiac surgery [5]. Serious complications may occur during catheterization as a result of PLSVC like shock, cardiac arrest, probably due to manipulation of the catheter in the CS, or perforation of the brachiocephalic vein if RSVC is absent [4]. Positioning a pacemaker lead through PLSVC and CS into right ventricle may be difficult in these patients $[6,7]$. If this is detected preoperatively, most of the operators prefer the left-sided approach, and if it comes out as on-table surprise while doing from the right side, it becomes technically challenging. Therefore, there is growing interest in tips and tricks about the pacemaker implantation 


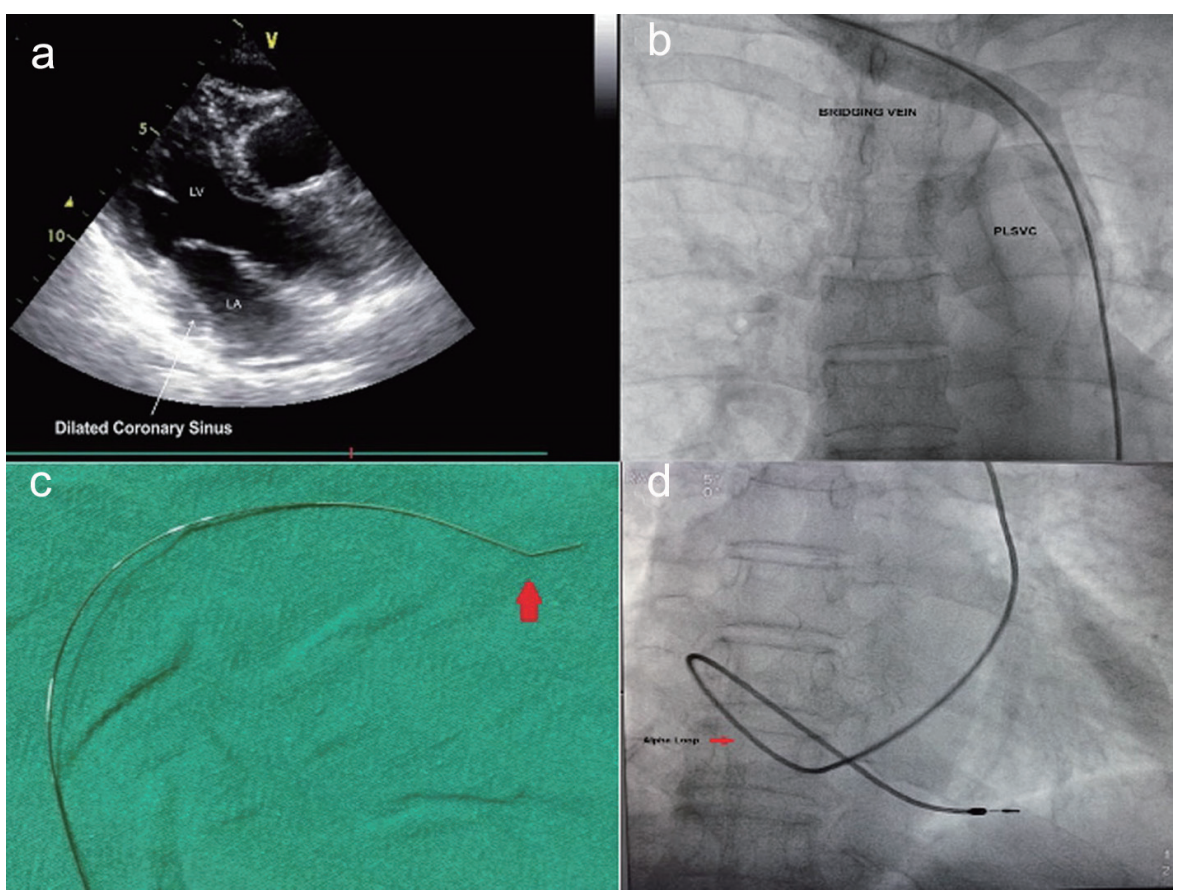

Figure 1. (a) TTE showing dilated coronary sinus. (b) Contrast phlebography from right upper limb showing isolated PLSVC. (c) Mond's curve. (d) Alpha loop configuration of ventricular lead (passive fixation lead).

from the right-sided approach in setting of isolated PLSVC.

\section{Materials and Methods}

\section{Aims}

The aim of the study was to assess technical challenges during pacemaker implantation, and their short- and long-term outcomes among patients with isolated PLSVC from right-sided approach in a prospective, single-centre study conducted in the Department of Cardiology, LPS Institute of Cardiology, G.S.V.M. Medical College, Kanpur, Uttar Pradesh, India from October 2008 to September 2018. Thirty-one consecutive patients with isolated PLSVC and 93 patients with RSVC were enrolled who presented with syncope with: 1) Sinus node dysfunction (SND); 2) Atrioventricular (AV) block, both congenital and acquired; and 3) Chronic bifascicular and trifascicular block with intermittent third-degree AV block, type 2 seconddegree AV block and alternating bundle branch block (BBB). There were 7,568 pacemaker implantations during the index period as it is the highest-level implantation performing centre in north India.

Enrolled patients underwent comprehensive evaluation which included clinical examination and investigations in the form of electrocardiogram, viral markers, electrolytes, hemogram and 2D transthoracic echocardiogram. If CS was found to be dilated (Fig. 1a), agitated saline contrast was injected from both right and left arms to look for its course and opacification of CS before right atrium (RA) to diagnose isolated PLSVC draining into RA (Table 1). Rarely, injection from left arm may opacify left atrium which signifies it as its draining site (Table 1). If diagnosed, preoperative contrast venography from right antecubital vein was done to demonstrate its course which served as a landmark during pacemaker implantation. Pacemakers were implanted as VVIR (ventricle paced, ventricle sensed, inhibited, and rate modulated), and DDDR (dualchamber paced, dual-chamber sensed, dual response to sensing (triggered and inhibited), and rate modulated).

\section{Pacemaker implantation and technique}

Informed consent was obtained from each patient. The implant site was prepared aseptically. Teicoplanin $400 \mathrm{mg}$ was given

Table 1. Echocardiographic Diagnosis of Isolated Persistent Left Superior Vena Cava and Its Possible Variation With Contrast Injection (Agitated Saline)

\begin{tabular}{lllll}
\hline Agitated saline contrast & RSVC & Isolated PLSVC draining to RA & PLSVC with RSVC & LSVC draining into LA \\
\hline Contrast from the left arm & RA & CS to RA & CS to RA & LA \\
Contrast from the right arm & RA & CS to RA & RA & RA \\
\hline
\end{tabular}

LA: left atrium; PLSVC: persistent left superior vena cava; RSVC: right superior vena cava; RA: right atrium; CS: coronary sinus. 


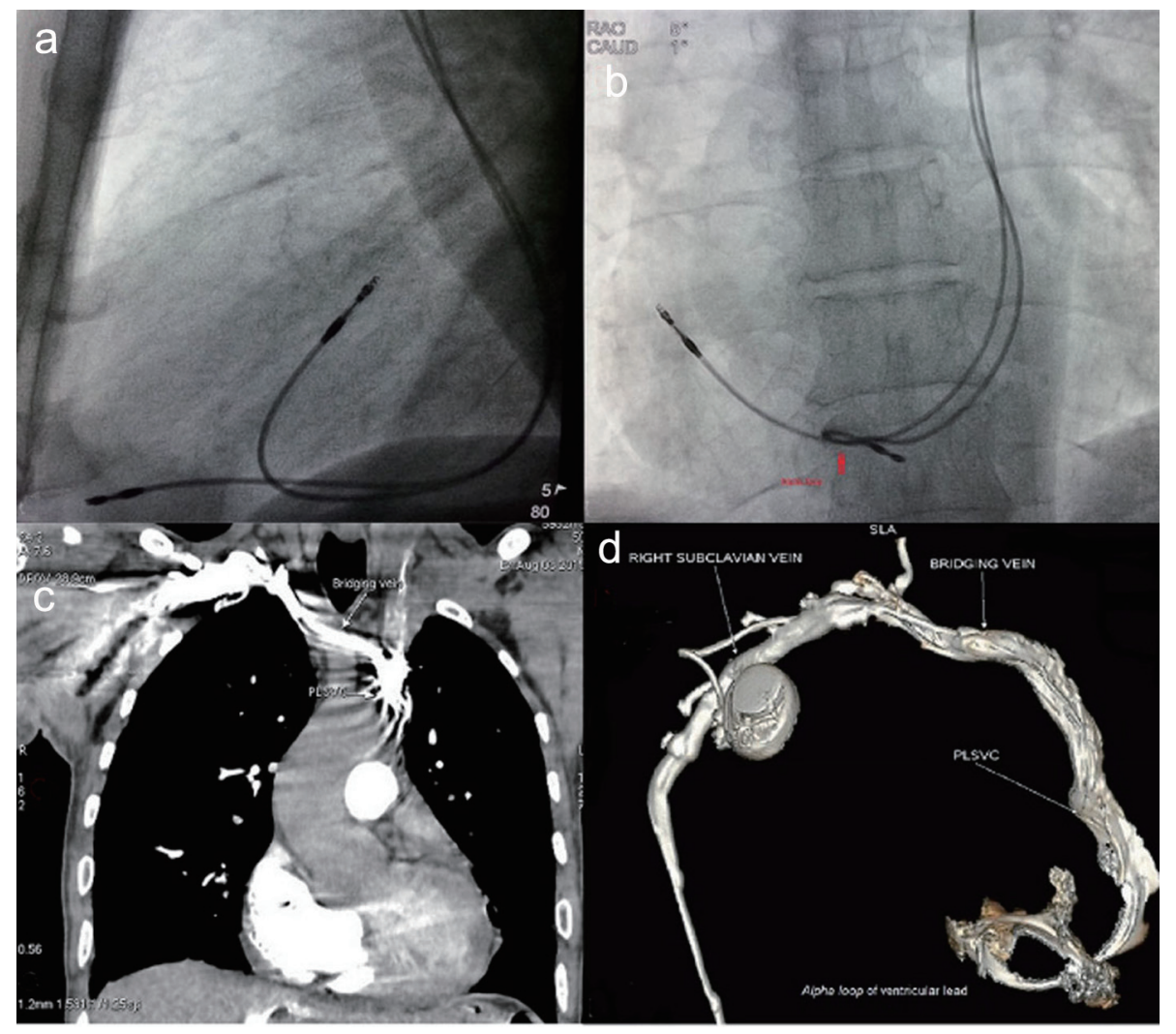

Figure 2. (a) Characteristic lead positions in a lateral view. (b) An antero-posterior view. (c) CT venography showing isolated PLSVC. (d) Multiple detector computed tomography (MDCT) showing isolated PLSVC.

45 min prior to skin incision. After puncture of right subclavian vein, pacing wire was manipulated to slip in left subclavian vein and to pass through left-sided superior vena cava (SVC). An $8 \mathrm{~F}$ pacemaker sheath was inserted by modified Seldinger technique. On lead insertion, circuitous course of the lead via left mediastinum instead of the expected right side was noted (Fig. 1a). Mond's curve (Fig. 1c) was introduced at the tip of stellate and introduced into pacing lead when septal pacing was planned. When reached the respective site, the lead was pushed a little while simultaneously pulling the metallic stellate in tandem thus providing characteristic alpha loop configuration (Fig. 1d). Lead position was confirmed in various projections (Fig. 2a, b) and it was screwed into ventricle once acceptable parameters were obtained. No such loop was needed for atrial positioning. Atrial lead was positioned either into interatrial septum or free wall (Fig. 2b). Once properly positioned, venous sheath was gradually pulled, and once outside the floor of muscle, it was peeled off. Multiple detector computed tomography (MDCT) and computed tomography (CT) venography were also done in a few initial cases to show a bridging vein draining the right jugular and right subclavian veins into PLSVC which was coursing on the left side of the mediastinum and left atrium before draining into the RA via a dilated CS (Fig. 2c, d). All patients received injection of teicoplanin $400 \mathrm{mg}$ twice daily for 2 days along with injection of amikacin $500 \mathrm{mg}$ once daily for 5 days as per our cath lab protocol. All patients were discharged on the sixth day and fol- lowed up serially every 3 months for the first year and then once annually.

\section{Statistical analysis}

Continuous variables were expressed as mean \pm SD. Categorical variables were expressed as percentages. Parametric continuous variables were compared by Student's $t$-test; categorical variables were compared by the Chi-square test. Study was designed on the basis of nested case-control method, and therefore 1:3 proportions was enrolment criteria to detect any difference as statistically significant as incidence of isolated PLSVC is low. P values $<0.05$ were considered significant. All statistical studies were carried out using the SPSS program (version 15.0; SPSS Inc, Chicago, IL, USA).

\section{Results}

\section{Baseline characteristics}

During the index period, a total of 7,568 permanent pacemaker (PM) implantations were performed by different operators, of which 31 patients had isolated PLSVC, while 93 patients with RSVC were enrolled. The youngest patient was 18 years old 
Table 2. Baseline Characteristics of Patients $(n=124)$

\begin{tabular}{llll}
\hline Baseline characteristics & PM-PLSVC $(\mathbf{n = 3 1 , \% )}$ & PM-without PLSVC (n= 93, \%) & P-value \\
\hline Age (years) & $65.3 \pm 11.6$ & $64.4 \pm 9.3$ & 0.6 \\
Sex (men/women) & $19 / 12$ & $57 / 36$ & 0.5 \\
HTN & $6(19 \%)$ & $22(24 \%)$ & 0.4 \\
DM & $5(16 \%)$ & $19(21 \%)$ & 0.23 \\
Ejection fraction (\%) & $62.5 \pm 4$ & $63.6 \pm 5$ & 0.34 \\
Pacing indication & & $42(45 \%)$ & 0.18 \\
$\quad$ SND & $13(44 \%)$ & $34(37 \%)$ & 0.16 \\
$\quad$ AV block & $05(12 \%)$ & $17(18 \%)$ & 0.2 \\
$\quad$ Chronic BFB and TFB & $09(29)$ & $36(39)$ & 0.4 \\
Types of pacemaker & $22(71)$ & $57(61)$ & 0.2 \\
$\quad$ VVI/VVIR & & $14(15)$ & 0.3 \\
$\quad$ DDD/DDDR & $05(16)$ & $79(85)$ & 0.19 \\
Types of pacing electrode & $26(84)$ & & \\
$\quad$ Tiened & Screwing & &
\end{tabular}

AV block: atrioventricular block; BFB: bifascicular block; DM: diabetes mellitus; EF: ejection fraction; HTN: hypertension; NYHA: New York Heart Association; PLSVC: persistent left superior vena cava; PM: pacemaker implantation; SND: sinus node dysfunction; TFB: trifascicular block.

while the eldest was 85 years old. Men outnumbered women (61\% vs. 39\%). There was no significant difference in the baseline parameters between both the groups. Syncope and palpitation were the most common symptoms. SND was the most common indication for pacemaker implantation $(n=55 ; 45 \%)$ (Table 2).

\section{Clinical outcome}

Mean procedural time was not significantly different although a little higher in PLSVC group as it required more manipulation from right-sided approach. There was no significant difference in mean fluoroscopic time, pacing parameters (threshold, impedance and amplitude) for atrial and ventricular leads, dislodgement rate, local site complication as minor hematoma, itching and pain, and follow-up duration between both groups. There was no incidence of subclavian crush syndrome in either group. Compared to patients with RSVC, all patients with PLSVC had alpha loop configuration for ventricular lead which was statistically significant ( 31 vs. $00 ; \mathrm{P}=0.002$ ) (Table 3).

\section{Discussion}

In early embryonic development, the venous blood of the upper part of the body drains into the RA via two bilaterally symmetrical running veins, i.e. left and right anterior cardinal veins $[3,4]$. Around 8 weeks of gestation, the left brachiocephalic vein develops as a bridge between the two cardinal veins and the part of the left anterior cardinal vein below this anastomosis usually collapses and degenerates, leaving only the right anterior cardinal vein which becomes anastomosed to the SVC. In about $20 \%$ of patients, the RSVC is absent resulting in drainage of venous blood from upper extremities through the left brachiocephalic vein, PLSVC and the CS into the RA, i.e. isolated PLSVC while in most patients RSVC is also present [3]. Persistent LSVC demonstrates several congenital variations including variable communication with RSVC, absence of right-sided SVC, drainage into the left atrium creating a right-to-left shunt, and is either isolated or in association with various anomalies [4]. If LSVC drains into left atrium either directly or in cases of unroofed CS, patients may be cyanotic. Such cases may be seen in association with atrial septal defect or other congenital cardiac anomaly. However, all of the patients in our study had isolated venous anomaly. Transvenous introduction of a lead from the RA to the right ventricle, through the $\mathrm{CS}$, becomes technically a demanding procedure in subjects with persistent LSVC, especially when the bridging innominate vein or right SVC is absent as seen in our study. The technical difficulties associated with persistent LSVC may lead to misplacement of catheter and injury to the vessel wall. Isolated PLSVC makes placement, manipulation and stability of pacing leads difficult as it takes acute angle after coming out of CS to cross the tricuspid valve, and to reach the RV apex. Despite this, the presence of a persistent LSVC does not necessarily preclude successful placement of a pacemaker or implantable cardioverter defibrillator (ICD) lead if it comes out as on-table surprise [8]. Since it takes a circuitous course, all ventricular leads had alpha loop compared to patients with RSVC in our study. That also added additional advantage in maintaining the lead stability as lead dislodgement rates were similar in both groups. Most of our patients had screwing lead but even those who had tined lead had similar stability over follow-up period. As right ventricle is a trabecu- 
Table 3. Clinical and Procedural Outcomes of Patients $(n=124)$

\begin{tabular}{|c|c|c|c|}
\hline Baseline characteristics & PM-PLSVC $(n=31, \%)$ & PM-without PLSVC $(\mathrm{n}=93, \%)$ & P-value \\
\hline Procedural time (min) & $25 \pm 11$ & $23 \pm 12$ & 0.24 \\
\hline Fluroscopic time (min) & $3.1 \pm 2.2$ & $2.7 \pm 2.1$ & 0.54 \\
\hline \multicolumn{4}{|l|}{ Length of leads (cm) } \\
\hline Atrial & 53 & 53 & NS \\
\hline Ventricular & 58 & 58 & NS \\
\hline Threshold (mV) & $1.2 \pm 0.1$ & $1 \pm 0.2$ & 0.4 \\
\hline P-wave (mV) & $4.1 \pm 0.2$ & $4.3 \pm 0.1$ & 0.5 \\
\hline Lead impedance $(\Omega)$ & $580 \pm 170$ & $540 \pm 140$ & 0.2 \\
\hline \multicolumn{4}{|l|}{ Ventricular lead } \\
\hline Alpha loop (ventricular lead) & $31(100)$ & 00 & 0.002 \\
\hline Lead dislodgement & $01(3.2)$ & $03(4.8)$ & 0.32 \\
\hline Subclavian crush & 00 & 00 & 0.00 \\
\hline Local site complication & $02(6.4)$ & $07(7.5)$ & 0.5 \\
\hline Follow-up duration (years) & $6.9 \pm 1.3$ & $7.2 \pm 1.1$ & 0.18 \\
\hline
\end{tabular}

PM: pacemaker implantation.

lated structure, lead with passive fixation also worked well as described by Kumar et al [9] with similar follow-up duration. Several authors have reported use of active fixation lead in this situation with similar results $[10,11]$.

Epicardial pacing may be another option in such scenario [12]. Our study demonstrated that endocardial pacing can be safely done in patients with isolated PLSVC from right-sided approach with excellent result over short and long terms. Endocardial implantation is found to be feasible even in small children with good results [13], though there were no small children in our group. Various options can be ventured for placement of RV endocardial lead as in our study. Changing the shape of the stylet once the lead reaches RA through CS was the most common one, helping to negotiate the lead across the tricuspid valve [3]. Abutting the lead on the free wall of RA gave a better support so that the lead could be pushed across the tricuspid valve. There are reports of using long $40-\mathrm{cm}$ worley sheath for better support for RV lead placement [14]. Also venous sheath can be left while manipulating the lead to enter the right ventricle. Initially straight stylet should be chosen as it makes its course little easier. Once it is there, stylet with Mond's modification is to be used if septal pacing is planned. Once acceptable parameters were obtained, venous sheath can be peeled by gradually pulling it and still keeping stylet there. It provides additional advantages as manipulation becomes much easier because it provides more support since the lead has to travel a long path to reach right ventricle. Once an adequate loop is visualized under fluoroscope, stylet can be withdrawn. In our study, length of atrial lead and ventricular lead were $53 \mathrm{~cm}$ and $58 \mathrm{~cm}$ as conventional leads though $\mathrm{Ku}-$ mar et al [9] and Rathakrishnan et al [15] had used a regular $58-\mathrm{cm}$ tined ventricular lead as an atrial electrode and fixed it in an atrial appendage to ensure an adequate sustained push against the RA wall. Many operators have reported use of active fixation lead with standard length for this situation [10, 11]. Although a little more manipulations are required for patients with PLSVC, total fluoroscopy time was not significantly different between both groups. In our study, all patients with isolated PLSVC had its drainage into RA, but had it been left atrium, surgical repair with epicardial lead placement would have been a better option.

\section{Conclusions}

Pre-procedural phlebography and contrast echo are extremely useful to identify these silent but challenging venous anomalies and to determine precisely the pathway to the RA. Even though permanent PM implantation through CS via PLSVC from right-sided approach is technically demanding, it obtains good long-term results. Left-sided approach, leadless pacemaker and epicardial implantation may be other options.

In our study, although the longest follow-up was 10 years, a few patients had shorter follow-up duration as well. Venous thrombosis, pacing failure, subclavian crush, and other possible complications were not seen till the follow-up duration (roughly 6 - 7 years). We believe that further extending followup to a longer period is needed (around 12 - 15 years). 


\section{Acknowledgments}

None.

\section{Financial Disclosure}

None.

\section{Conflict of Interest}

None.

\section{Informed Consent}

Informed written consent was taken from patients.

\section{Author Contributions}

None.

\section{References}

1. Danielpour PJ, Aalberg JK, El-Ramey M, Sivina M, Wodnicki $H$. Persistent left superior vena cava: an incidental finding during central venous catheterisation-a case report. Vasc Endovascular Surg. 2005;39:109-111.

2. Palinkas A, Nagy E, Forster T, Morvai Z, Nagy E, Varga A. A case of absent right and persistent left superior vena cava. Cardiovasc Ultrasound. 2006;4:6.

3. Peltier J, Destrieux C, Desme J, Renard C, Remond A, Velut S. The persistent left superior vena cava: anatomical study, pathogenesis and clinical considerations. Surg Radiol Anat. 2006;28(2):206-210.

4. Tak T, Crouch E, Drake GB. Persistent left superior vena cava: incidence, significance and clinical correlates. Int J Cardiol. 2002;82(1):91-93.

5. Chan KL, Abdulla A. Images in cardiology. Giant coronary sinus and absent right superior vena cava. Heart. 2000;83(6):704.
6. Schummer W, Schummer C, Frober R. Persistent left superior vena cava and central venous catheter position: clinical impact illustrated by four cases. Surg Radiol Anat. 2003;25(3-4):315-321.

7. Golzio PG, Franco E, Chiribiri A. Atrio-ventricular synchronization by single VDD lead inserted through persistent left superior vena cava in patient with Turner's syndrome. Pacing Clin Electrophysiol. 2006;29(10):11811182.

8. Beukema RJ, Elvan A, Beukema WP, Misier AR, Delnoy PP, Luttikhuis HA, Hoorntje JC. Cardioverter defibrillator implantation in a patient with absent right superior vena cava. Neth Heart J. 2006;14(7-8):255-257.

9. Kumar S, Moorthy N, Kapoor A, Sinha N. A challenging dual chamber permanent pacemaker implantation in persistent left superior vena cava with absent right superior vena cava. J Cardiol Cases. 2012;5(2):e122-e124.

10. Roberts DH, Bellamy CM, Ramsdale DR. Implantation of a dual chamber pacemaker in a patient with persistent left superior vena cava. Int J Cardiol. 1992;36(2):242243.

11. Zardo F, Nicolosi GL, Burelli C, Zanuttini D. Dual-chamber transvenous pacemaker implantation via anomalous left superior vena cava. Am Heart J. 1986;112(3):621622.

12. Lenox CC, Zuberbuhler JR, Park SC, Neches WH, Mathews RA, Fricker FJ, Bahnson HT, et al. Absent right superior vena cava with persistent left superior vena cava: implications and management. Am J Cardiol. 1980;45(1):117-122.

13. Dalili M, Alizadeh A, Haghjoo M. Successful Implantation of Transvenous Pacing System via Persistent Left Superior Vena Cava and Coronary Sinus in Small Children. Indian Pacing Electrophysiol J. 2011;10(12):551555.

14. Mitov V, Perisic Z, Kostic T, Stojkovic A, Jolic A, Aleksic A, Milasinovic G. An alternative approach for endocardial pacemaker lead implantation in patient with persistent left superior vena cava. Srp Arh Celok Lek. 2010;138(12):85-87.

15. Rathakrishnan SS, Kaliappan T, Gopalan R. Beware of venous anomalies in young patients with sick sinus syndrome: a report of two cases of sick sinus syndrome with systemic venous anomalies. Heart Views. 2015;16(3):107-110. 